\title{
ENDEMISMO REGIONAL PRESENTE EN LA FLORA DEL MUNICIPIO DE CULIACÁN, SINALOA, MÉXICO
}

\author{
Rito Vega-Aviña, Hipólito Aguiar-Hernández, \\ Juan Antonio Gutiérrez-García, Jorge Alejandro Hernández-Vizcarra \\ Facultad de Agronomía \\ Universidad Autónoma de Sinaloa \\ Apartado postal 726 \\ 80000 Culiacán, Sinaloa \\ InÉs Fernando Vega-LópeZ \\ Escuela de Informática \\ Universidad Autónoma de Sinaloa \\ Prolongación Josefa Ortiz de Domínguez s/n \\ Col. La Lima, Culiacán, Sinaloa \\ Y \\ JosÉ LUIS VILLASEÑOR \\ Instituto de Biología, Departamento de Botánica \\ Universidad Nacional Autónoma de México \\ Apartado postal 70-367 \\ 04510 México, D.F.
}

\begin{abstract}
RESUMEN
El municipio de Culiacán, con un área de $4758.9 \mathrm{~km}^{2}$, se caracteriza por su topografía relativamente plana, su baja elevación altitudinal $(0-300 \mathrm{~m})$ y pocos accidentes montañosos que sobrepasan los $500 \mathrm{~m}$. El principal tipo de vegetación es el bosque espinoso; sin embargo, también se encuentran manglar, bosque tropical caducifolio, bosque tropical subcaducifolio, bosque de Quercus y bosque de Pinus y Quercus. La flora vascular del municipio de Culiacán, Sinaloa, registra la presencia de 1445 especies y taxa subespecíficos distribuidos en 623 géneros y 145 familias. 162 taxa endémicos a la región oeste y noroeste de México están presentes en el área de estudio y dos de ellos con área restringida al municipio. Se discuten los patrones de distribución de los endemismos en los estados que conforman tal región mexicana.
\end{abstract}

\section{ABSTRACT}

The municipio of Culiacán has an area of $4758.9 \mathrm{~km}^{2}$ and is located in the central portion of the state of Sinaloa. Its topography is relatively flat, with a large percentage of its surface occurring 
below $300 \mathrm{~m}$ of altitude and only few places surpass $500 \mathrm{~m}$ above sea level. The main vegetation type is the thorn forest; however, the mangrove forest, the tropical deciduous forest, the tropical subdeciduous forest, the Quercus forest, and the Pinus and Quercus forest are also present although in less proportion. An inventory of the vascular flora recorded 1445 native species and subspecific taxa, in 623 genera and 145 families. 162 endemic taxa to western (W) and northwestern (NW) regions of Mexico are present in the municipio of Culiacán, two of them being restricted to its political borders. In this paper, a checklist of the endemic taxa to W and NW Mexico occurring in the municipio is presented; their distribution patterns at state level in the region are also discussed.

\section{INTRODUCCIÓN}

Según Rzedowski (1991b) y Toledo (1994), la zona de máxima concentración de flora vascular se extiende en México de Chiapas a Oaxaca y de ahí se bifurca en dos franjas, que van disminuyendo su diversidad a medida que se alejan hacia el norte. Una se dirige hacia el centro de Veracruz y la otra rumbo a Sinaloa y Durango. Lamentablemente la diversidad de esta última franja occidental está aún mal conocida; sobre todo en lo que toca a la Sierra Madre Occidental, o más específicamente el estado de Sinaloa y partes de Chihuahua, Sonora, Durango y Nayarit (Cabrera-Rodríguez y Villaseñor, 1987; Dávila, 1992 y Chiang, 1993). La falta de estudios botánicos en la región sigue siendo un factor determinante que limita las estimaciones reales de la riqueza florística presente.

La variación fisiográfica y climática manifestada por la presencia de la zona costera al oeste, la serranía al noreste, el desierto al norte, y la zona húmeda al sur, así como la variable altitudinal de 0-2 $710 \mathrm{~m}$ s.n.m., hacen de Sinaloa un área con variados hábitats. Además, la presencia de la Provincia de la Sierra Madre Occidental de la Región Mesoamericana de Montaña con influencia del Reino Holártico, la Provincia de la Planicie Costera del Noroeste de la Región Xerofítica Mexicana y la Provincia de la Costa Pacífica de la Región Caribea del Reino Neotropical (Rzedowski, 1978), indudablemente propician la presencia de una riqueza vegetal que, aunque todavía no es conocida con precisión, Vega et al. (1989) la estiman en alrededor de 3500 especies de plantas vasculares.

En el municipio de Culiacán están en contacto dos provincias florísticas, la Planicie Costera del Noroeste y la Costa Pacífica. Por lo tanto, es de esperarse una relación con floras afines como la del desierto de Sonora y de áreas tropicales, según los criterios de riqueza y endemismos que señala Rzedowski (1991a, 1991b).

Son pocos los trabajos precedentes que permiten estimar la riqueza florística del estado y en particular la del municipio de Culiacán. Entre los más importantes se pueden citar los de Brandegee (1905, 1908), Ponce de León (1909), Riley (1923, 1924), González O. (1927, 1929), Quintanar (1938), Gentry $(1940,1946,1948)$ y Sánchez (1961). En fechas relativamente recientes el estado de Sinaloa ha sido objeto de trabajos sistemáticos encaminados a inventariar su diversidad vegetal. Entre los primeros resultados se pueden mencionar el recuento de la Flora de Sinaloa (Vega et al., 1989), las malezas del Valle de Culiacán (Bojórquez y Vega, 1989), el estudio florístico de la Península de Lucenilla (Hernández y Vega, 1989) y la flora de la Isla Venados (Flores et al., 1996). Por otra parte, se han iniciado los estudios taxonómicos de algunos grupos vegetales presentes en la entidad (Vega, 1991, 1992). Un recuento actualizado, aunque preliminar de su flora, arroja la cifra de 2792 especies de plantas vasculares, distribuidas en 978 géneros y 202 familias (Cuadro 1). 
Vega-Aviña et al.: Endemismo Regional en la Flora del Municipio de Culiacán, Sinaloa

Cuadro 1. Riqueza de plantas vasculares registradas para el estado de Sinaloa (entre paréntesis) y el municipio de Culiacán.

\begin{tabular}{|l|c|c|c|}
\hline Grupo taxonómico & Familias & Géneros & Especies \\
\hline Pteridophyta & $8(15)$ & $13(39)$ & $32(120)$ \\
Gymnospermae & $2(4)$ & $2(6)$ & $2(19)$ \\
Liliopsida (Monocotyledoneae) & $22(28)$ & $91(142)$ & $205(359)$ \\
Magnoliopsida (Dicotyledoneae) & $113(155)$ & $517(791)$ & $1206(2294)$ \\
TOTAL & $145(202)$ & $623(978)$ & $1445(2792)$ \\
\hline
\end{tabular}

Con el propósito de contribuir al conocimiento de la flora de Sinaloa, en 1994 se inicia el estudio del municipio de Culiacán. Considerando que los criterios de riqueza de especies y endemismos han sido los dos argumentos principales para recomendar la selección de áreas naturales protegidas (Margules y Usher, 1981; Götmark y Nilsson, 1992), el presente trabajo tiene como objetivo la elaboración de una lista florística de los taxa endémicos al oeste y noroeste de México que están presentes en el área de estudio, así como el análisis de sus patrones de distribución en los estados que conforman la región y tipo de vegetación donde se han detectado. Tal región, arbitrariamente constituida desde el punto de vista florístico, la integran los estados de la Costa del Pacífico, desde Baja California hasta Colima, además de los correspondientes a Chihuahua, Durango y Zacatecas.

\section{ÁREA DE ESTUDIO}

El municipio de Culiacán se encuentra situado en la porción central del estado de Sinaloa, entre los $24^{\circ} 02^{\prime} 10^{\prime \prime}$ y $25^{\circ} 14^{\prime} 56^{\prime \prime}$ de latitud N y los $106^{\circ} 56^{\prime} 50^{\prime \prime}$ y $107^{\circ} 50^{\prime} 15^{\prime \prime}$ de longitud W (Fig. 1). Con una extensión de $4758.9 \mathrm{~km}^{2}$, ocupa $8.2 \%$ de la superficie del estado de Sinaloa. El municipio colinda hacia el suroeste con el Golfo de California. Las corrientes hídricas principales son los ríos Humaya, Tamazula, Culiacán y San Lorenzo. La mayor parte de su superficie tiene una altitud sobre el nivel del mar que oscila entre 0 y 500 m (Fig. 1). Las elevaciones que sobrepasan esta cota son reducidas en superficie. Sobresalen en altitud el Cerro Prieto (840 m s.n.m.), el Cerro Los Laureles (840 m s.n.m.), el Cerro La Mojonera (760 m s.n.m.) y el Cerro El Pinito (860 m s.n.m.). La porción costera está formada por planicies no mayores de $40 \mathrm{~m}$ sobre el nivel del mar y por costas de emersión, resultado de la aparición de una parte de la plataforma continental que ha surgido por el descenso del nivel del mar (Anónimo, 1985; Anónimo, 1994).

Las características geológicas del municipio se pueden clasificar en tres grupos básicos: 1) la faja costera, formada por capas recientes del Pleistoceno y por formaciones de principios del Cuaternario; 2) la región central, formada por rocas volcánicas del Cenozoico y 3) las partes elevadas de la sierra, formadas por rocas metamórficas del 


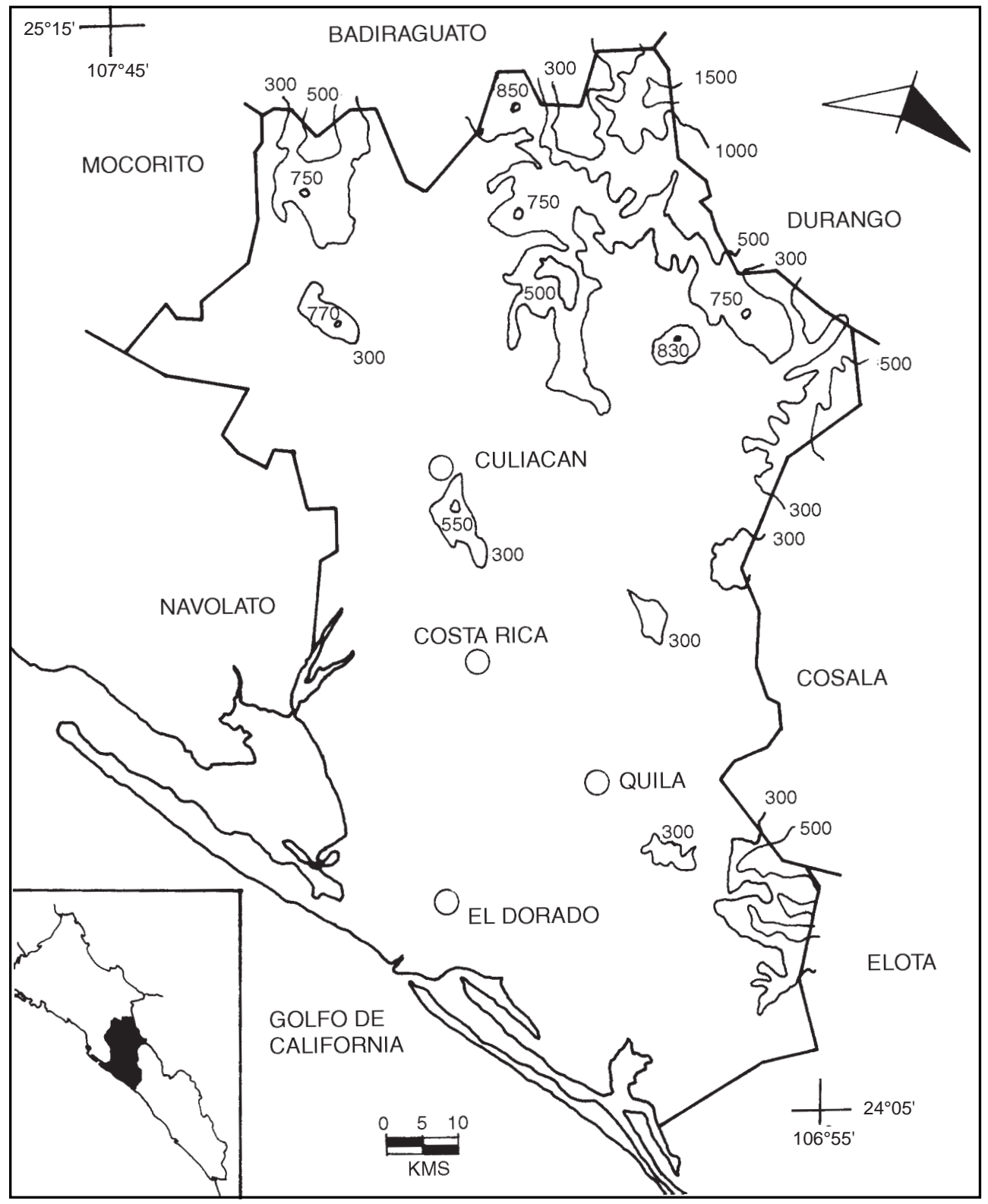

Fig. 1. Localización y topografía del municipio de Culiacán, Sinaloa. 
Vega-Aviña et al.: Endemismo Regional en la Flora del Municipio de Culiacán, Sinaloa

Cenozoico. Entre los tipos de suelos se han registrado los feozem háplicos, los cambisoles, los litosoles, los fluvisoles, los regosoles, los vertisoles y los solonchaks (Anónimo,1985).

En el municipio de Culiacán se identifican dos grupos principales de climas (García, 1973): los secos $\left(B S_{0}, B S\right)$ y los cálidos $\left(\mathrm{Aw}_{0}, \mathrm{Aw}\right)$. En la parte costera y en la porción media del municipio prevalecen los primeros y en las zonas altas se presentan los segundos. La temperatura promedio anual varía de la costa a las zonas altas de 23 a $26^{\circ} \mathrm{C}$, igualmente, la precipitación promedio anual varía de los 400 a los 800 mm. (Anónimo, 1985; Rendón, 1995).

El bosque espinoso es el principal tipo de vegetación en el municipio (Rzedowski, 1978). Cerca de la costa se encuentran manglares y vegetación halófila. En lugares sombreados o cañadas es posible hallar manchones de bosque tropical caducifolio o bosque tropical subcaducifolio, representado básicamente por Brosimum alicastrum y Ficus spp. En elevaciones superiores a los $400 \mathrm{~m}$ se encuentran pequeños bosques de Quercus y en la región noreste del municipio, en altitudes mayores de $700 \mathrm{~m}$ se encuentran sitios con bosque mixto de Pinus oocarpa y Quercus spp. A lo largo de algunos arroyos se desarrolla el bosque de galería, dominado por Taxodium mucronatum y a la orilla de los ríos es sobresaliente la presencia de Populus mexicana subsp. dimorpha y Salix nigra.

\section{METODOLOGÍA}

El listado florístico para la zona de estudio se elaboró con base en aproximadamente 2800 colectas previas de plantas vasculares depositadas en los herbarios de la Facultad de Agronomía y de la Escuela de Biología, ambas de la Universidad Autónoma de Sinaloa, y en 5435 ejemplares obtenidos en 320 sitios, entre enero de 1994 y enero de 1997. Por razones de seguridad, no se recolectó en las partes altas de la Sierra de Tacuichamona y de la Sierra Chanteco, ubicadas al SE y NE del municipio.

La determinación de los ejemplares herborizados se hizo mediante la consulta de literatura botánica especializada, del estudio del material depositado en el Herbario Nacional (MEXU) del Instituto de Biología de la Universidad Nacional Autónoma de México (UNAM) y de la Universidad Autónoma de Sinaloa (UAS), así como con la colaboración de los taxónomos del Instituto de Biología de la UNAM. El sistema de clasificación usado para el arreglo de las especies de la clase Liliopsida (Monocotiledóneas) es el formulado por Dahlgren et al. (1985) y para la clase Magnoliopsida (Dicotiledóneas) el propuesto por Cronquist (1981). Los autores de los nombres científicos están citados de acuerdo con Brummitt y Powell (1992).

Para determinar el elemento endémico presente en la región se analizaron los patrones de distribución geográfica de los taxa registrados. En este trabajo se consideran endémicas aquellas especies cuya área no excede los límites del territorio oeste y noroeste de México. Para definir los patrones de distribución de las especies, se revisaron los trabajos que documentan la flora de esta región (Brandegee, 1905, 1908; Ponce de León, 1909; Riley, 1923, 1924; González O., 1927, 1929; Quintanar, 1938; Gentry, 1940, 1942, 1946; LeSueur, 1945; White, 1948; Shreve y Wiggins, 1964; Wiggins, 1980; Vega et al., 1989; González E. et al., 1991, 1993; Laferriere, 1994; y Téllez, 1995), revisiones taxonómicas, estudios florísticos de otras regiones tanto de México como de Estados Unidos y Centro 
y Sudamérica. Todo ello se complementó con consultas al acervo del material depositado en el Herbario Nacional (MEXU).

\section{RESULTADOS}

La riqueza de plantas vasculares del municipio se estima en 1445 especies repartidas en 623 géneros y 145 familias (Cuadro 1). En el área de estudio están representadas $71.8 \%$ de las familias, $63.7 \%$ de los géneros y $51.7 \%$ de las especies registradas para el estado de Sinaloa.

Considerando a las especies y taxa subespecíficos, 353 de ellos son endémicos a México y 162 son endémicos a la porción oeste y noroeste de México. El endemismo está repartido en 49 familias, sobresaliendo por su número Asteraceae (26), Cactaceae (19), Euphorbiaceae (14), Fabaceae (13) y Mimosaceae (8). Echeveria kimnachii Meyrán et Vega (Crassulaceae) y Hofmeisteria sinaloensis Gentry (Asteraceae) sólo se conocen dentro de los límites políticos del municipio. Una probable nueva especie de Cunila (Lamiaceae) incrementaría a tres este endemismo estricto.

En el estado de Sinaloa se registraron 383 especies y taxa subespecíficos endémicos de la región oeste y noroeste de México. De ellos, 107 son endémicos estrictos al estado y 21 están presentes en el municipio, lo que representa $20 \%$ del endemismo estricto estatal. El análisis de los patrones de distribución geográfica de las especies indica una mayor similitud florística con los estados de Nayarit (932), Veracruz (813) y Sonora (708).

Con Sonora el municipio comparte el mayor número de endemismos regionales (86, 18 de ellos endémicos estrictos de Sonora y Sinaloa). Le siguen por su número Nayarit (64, 11 de ellos endémicos estrictos a ambos estados), Jalisco (52 y 6), Chihuahua (38 y 3), Durango (35 y 4), Baja California Sur (23 y 1), Colima (12 y 1), Baja California (9) y Zacatecas (2).

El bosque espinoso registró el mayor número de endemismos (126, 72 de ellos restringidos a este tipo de vegetación). Le siguen en importancia el bosque tropical caducifolio (52, 8 de ellos exclusivos a este tipo de vegetación), el bosque de Quercus (32 y 18), el bosque tropical subcaducifolio (28 y 5), el manglar (11) y la vegetación acuática y subacuática (4).

\section{DISCUSIÓN}

Los resultados indican que el municipio de Culiacán no despunta por su riqueza endémica local. Sin embargo, a nivel regional sí tiene un lugar sobresaliente, tanto en endemismo como en riqueza de especies. Con la mitad de la flora estatal y del endemismo regional, el área de estudio destaca como un sitio importante para la conservación de la flora vascular del oeste y noroeste del país.

El municipio presenta una mayor similitud florística con estados cuyo territorio se ubica en la Región Florística Caribea (Rzedowski, 1978), aunque comparte un mayor número de endemismos con Sonora, circunscrito mayormente en la Región Florística Xerofítica Mexicana. Estos datos apoyan las observaciones de que la riqueza de la flora está 
Vega-Aviña et al.: Endemismo Regional en la Flora del Municipio de Culiacán, Sinaloa

especialmente asociada a sitios con mayor humedad y el endemismo tiene una más estrecha relación con las zonas secas.

La mayoría de las especies endémicas mencionadas en este trabajo son elementos característicos del bosque espinoso (78\%). Lo anterior es indicativo de que en esta comunidad vegetal ha evolucionado un importante número de elementos autóctonos, muy seguramente a lo largo de la historia y conformación de este tipo de vegetación. Desafortunadamente el bosque espinoso mexicano es uno de los tipos de vegetación menos representado en las áreas naturales protegidas de nuestro país (Anónimo, 1996) y al que menos atención se le ha puesto.

El endemismo representa la rareza de cualquier flora. En el municipio de Culiacán se ha detectado un importante porcentaje $(11 \%)$ de elementos exclusivos a la región oeste y noroeste de México, que al parecer no están presentes en las áreas naturales protegidas y que carecen de algún programa de protección. Creemos que aunque la región presenta una alta tasa de transformación de las comunidades naturales, sobre todo por las actividades humanas, todavía es posible establecer estrategias para su conservación. La compilación de datos como los aquí presentados es una etapa básica hacia la consolidación de un programa mejor organizado para la conservación a largo plazo de esta rareza.

\section{AGRADECIMIENTOS}

Este trabajo fue realizado gracias al financiamiento de la Comisión Nacional para el Conocimiento y Uso de la Biodiversidad (CONABIO), a través del proyecto "Flora del municipio de Culiacán, Sinaloa", según convenio B022, conducido entre 1994 y 1995 por el primer autor en la Facultad de Agronomía de la UAS. Los autores desean expresar su agradecimiento a F. Hernández A., G. A. Bojórquez B., M. Aguilar P., J. S. Palazuelos N. y R. M. Esquerra V. por su valiosa colaboración en la recolección y determinación del material botánico. De igual manera se agradece a P. Dávila A., R. Lira S., O. Téllez V., F. Chiang C., F. Ramos M., A. Delgado S., M. García P., I. Méndez L., S. Arias G. y A. García M. por su importante apoyo en la determinación del material recolectado.

\section{LITERATURA CITADA}

Anónimo. 1985. Municipio de Culiacán, síntesis monográfica. Dirección de Estadística y Estudios Económicos. Secretaría de Hacienda Pública y Tesorería. Gobierno del Estado de Sinaloa. Culiacán, Sinaloa. 13 pp.

Anónimo. 1994. Cuaderno estadístico municipal de Culiacán, estado de Sinaloa. Instituto Nacional de Estadística, Geografía e Informática, Gobierno del Estado de Sinaloa y H. Ayuntamiento Constitucional de Culiacán. Talleres Gráficos del Instituto Nacional de Estadística, Geografía e Informática. Aguascalientes, Aguascalientes. $131 \mathrm{pp}$.

Anónimo. 1996. Programa de áreas naturales protegidas de México 1995-2000. Secretaría del Medio Ambiente, Recursos Naturales y Pesca. Instituto Nacional de Ecología. México, D.F. 138 pp.

Bojórquez B., G. A. y R. Vega. 1989. Malezas del Valle de Culiacán. Secretaría de Educación Pública - Universidad Autónoma de Sinaloa. Culiacán, Sinaloa. 18 pp.

Brandegee, T. S. 1905. Plants from Sinaloa, Mexico. Zoe 5: 196-226.

Brandegee, T. S. 1908. Plants from Sinaloa, Mexico. Zoe 5: 241-244. 
Brummitt, R. K. y C. E. Powell (eds.) 1992. Authors of plant names. Royal Botanic Gardens, Kew. 732 $\mathrm{pp}$.

Cabrera-Rodríguez, L. y J. L. Villaseñor R. 1987. Revisión bibliográfica sobre el conocimiento de la familia Compositae en México. Biótica 12: 135-138.

Chiang C., F.1993. Plantas vasculares. In: Guevara, S., P. Moreno-Casasola y J. Rzedowski (comps.). Logros y perspectivas del conocimiento de los recursos vegetales de México en vísperas del siglo XXI. Instituto de Ecología, A.C. y Sociedad Botánica de México, A.C. México, D.F. pp. 75-84.

Cronquist, A. 1981. An integrated system of classification of flowering plants. Columbia University Press. Nueva York. 1262 pp.

Dahlgren, R. M. T., H. T. Clifford y P. F. Yeo. 1985. The families of the monocotyledons: structure, evolution and taxonomy. Springer-Verlag. Berlin. $520 \mathrm{pp}$.

Dávila A., P. D. 1992. Un análisis de los herbarios mexicanos. Ciencias. Número especial 6: 57-60.

Flores C., L. M., R. Vega A., D. Benítez P. y F. Hernández A. 1996. Flora de la Isla Venados de Bahía Mazatlán, Sinaloa, México. Anales Inst. Biol. Univ. Nac. México, Ser. Bot. 67: 283-301.

García, E. 1973. Modificaciones al sistema de clasificación climática de Köppen (para adaptarlo a las condiciones de la República Mexicana). 2a. edición. Instituto de Geografía, Universidad Nacional Autónoma de México. México, D.F. 246 pp.

Gentry, H. S. 1940. Sierra Tacuichamona - a Sinaloa plant locale. Bull. Torrey Bot. Club 73: 356-362.

Gentry, H. S. 1942. Rio Mayo plants. Publ. Carnegie Inst. Wash. 527: 5-330.

Gentry, H. S. 1946. Notes on the vegetation of Sierra Surutato in Northern Sinaloa. Bull. Torrey Bot. Club 73: 351-462.

Gentry, H. S. 1948. Additions to the flora of Sinaloa and Nuevo Leon. Brittonia 6: 329-331.

González E., M., S. González E. y Y. Herrera A. 1991. Flora de Durango. In: Listados Florísticos de México IX. Instituto de Biología, Universidad Nacional Autónoma de México. México, D.F. $167 \mathrm{pp}$.

González E., S., M. González E. y A. Cortés O. 1993. Vegetación de la reserva de la biósfera La Michilía, Durango, México. Acta Bot. Mex. 22: 1-104.

González O., J. 1927. Exploración biológica por la Cuenca del Río Tamazula. Mem. Soc. Cient. Antonio Alzate 47: 145-153.

González O., J. 1929. Catálogo sistemático de las plantas de Sinaloa. Imprenta de la Escuela Preparatoria de Mazatlán. Mazatlán, Sinaloa. 50 pp.

Götmark, F. y C. Nilsson. 1992. Criteria used for protection of natural areas in Sweden 1909-1986. Conserv. Biol. 6: 220-231.

Hernández A., F. y R. Vega A. 1989. Flora de la Península de Lucenilla. Secretaría de Educación Pública - Universidad Autónoma de Sinaloa. Culiacán, Sinaloa. 24 pp.

Laferriere, J. E. 1994. Vegetation and flora of the Mountain Pima Village of Nabogame, Chihuahua, Mexico. Phytologia 77: 102-140.

LeSueur, H. 1945. The ecology of the vegetation of Chihuahua, Mexico, north of parallel twenty-eight. The University of Texas Publication No. 4521. Austin, Texas. 92 pp.

Margules, C. R. y M. B. Usher. 1981. Criteria used in assessing wildlife conservation potential: a review. Biol. Conserv. 21: 79-109.

Ponce de León, R. 1909. Ligeros apuntes sobre la flora del estado de Sinaloa. Talleres Tipográficos de Julio G. Arce. Culiacán, Sinaloa. 20 pp.

Quintanar, F. 1938. Estudios agrícolas de Sinaloa. Secretaría de Agricultura y Fomento - Confederación de Asociaciones Agrícolas del Estado de Sinaloa. Culiacán, Sinaloa. 25 pp.

Rendón V., D. 1995. Culiacán: en el umbral de una nueva imagen. H. Ayuntamiento de Culiacán Coordinación General de Turismo del Estado de Sinaloa - Instituto Tecnológico y de Estudios Superiores de Monterrey. Culiacán, Sinaloa. $137 \mathrm{pp}$.

Riley, L. A. M. 1923. Contributions to the flora of Sinaloa: I-IV. Bull. Misc. Inform. 3: 103-115; 4: 163175; 9: 333-346; 10: 388-401. 
Riley, L. A. M. 1924. Contributions to the flora of Sinaloa: V. Bull. Misc. Inform. 5: 206-222.

Rzedowski, J. 1978. Vegetación de México. Editorial Limusa. México, D.F. 423 pp.

Rzedowski, J. 1991a. Diversidad y orígenes de la flora de México. Acta Bot. Mex. 14: 3-21.

Rzedowski, J. 1991b. El endemismo en la flora fanerogámica mexicana: una apreciación analítica preliminar. Acta Bot. Mex. 15: 47-64.

Sánchez M., H. 1961. Las cactáceas del estado de Sinaloa. Cact. Suc. México 6: 27-39.

Shreve, F. e I. L. Wiggins. 1964. Vegetation and flora of the Sonoran Desert. Stanford University Press. Stanford, California. Vols. I y II. 1740 pp.

Téllez V., O. 1995. Flora, vegetación y fitogeografía de Nayarit, México. Tesis, Maestría en Ciencias. Facultad de Ciencias, Universidad Nacional Autónoma de México. México, D. F. 166 pp.

Toledo M., V. M. 1994. La diversidad biológica de México. Ciencia y Desarrollo 14: 17-30.

Vega A., R. 1991. Platanaceae y Taxodiaceae. In: Gálvez R., J. B. y C. Hernández R. (eds.). Agronomía en Sinaloa 3 (suplemento 1). Secretaría de Educación Pública - Universidad Autónoma de Sinaloa. Culiacán, Sinaloa. 8 pp.

Vega A., R. 1992. Simaroubaceae y Hernandiaceae. In: Gálvez R., J. B. y C. Hernández R. (eds.). Agronomía en Sinaloa 4 (suplemento 2). Secretaría de Educación Pública - Universidad Autónoma de Sinaloa. Culiacán, Sinaloa. 8 pp.

Vega A., R., G. A. Bojórquez B. y F. Hernández A. 1989. Flora de Sinaloa. Secretaría de Educación Pública-Universidad Autónoma de Sinaloa. Culiacán, Sinaloa. 49 pp.

White, S. S. 1948. The vegetation and flora of the region of the Río de Bavispe in Northeastern Sonora, Mexico. Lloydia 11: 229-302.

Wiggins, I. L. 1980. Flora of Baja California. Stanford University Press. Stanford, California. 1025 pp. 


\section{APÉNDICE}

Lista de especies endémicas a la región oeste y noroeste de México presentes en el municipio de Culiacán, Sinaloa. Se incluyen estados y tipos de vegetación donde se distribuyen. BC= Baja California, $\mathrm{BCS}=$ Baja California Sur, $\mathrm{Chih}=$ Chihuahua, Col= Colima, Jal= Jalisco, Nay= Nayarit, $\mathrm{Sin}=$ Sinaloa, $\mathrm{Son}=$ Sonora, $\mathrm{ZaC}=$ Zacatecas; $\mathrm{AR}=$ arvenses, $\mathrm{BE}=$ bosque espinoso, $\mathrm{BQ}=$ bosque de Quercus, $\mathrm{BTC}=$ bosque tropical caducifolio, $\mathrm{BTS}=$ bosque tropical subcaducifolio, $\mathrm{M}=$ manglar, $\mathrm{VAS}=$ vegetación acuática y subacuática.

\section{CLASE LILIOPSIDA}

Agavaceae

Agave bovicornuta Gentry [CHIH, SIN, SON] (BQ)

Manfreda singuliflora (S. Watson) Rose [CHIH, DGO, SIN, ZAC] (BQ)

Arecaceae

Brahea aculeata (Brandegee) H. E. Moore [NAY, SIN, SON] (BTC)

Bromeliaceae

Tillandsia exserta Fernald [SIN, SON] (M, BE)

Poaceae

Chloris brandegeei (Vasey) Swallen [BC, BCS, SIN] (BE, AR)

Muhlenbergia elongata Beal [CHIH, SIN, SON] (BQ)

\section{CLASE MAGNOLIOPSIDA}

Acanthaceae

Dyschoriste novogaliciana T. F. Daniel [DGO, JAL, NAY, SIN] (BQ)

Ruellia leucantha Brandegee var. postinsularis (Gentry) T. F. Daniel [SIN] (BE)

Amaranthaceae

Achyranthes watsonii Standl. [CHIH, SIN, SON] (BE)

Amaranthus lepturus S. F. Blake [BC, BCS, SIN] (BE)

Amaranthus watsonii Standl. [BC, BCS, SIN, SON] (BE)

Iresine hartmanii Uline [CHIH, DGO, JAL, SIN, SON] (BE)

Aristolochiaceae

Aristolochia taliscana Hook. \& Arn. [JAL, NAY, SIN] (BE)

Asclepiadaceae

Asclepias leptopus I. M. Johnst. [CHIH, SIN, SON] (BE)

\section{Asteraceae}

Adenophyllum anomalum (Canby \& Rose) Strother [DGO, NAY, SIN, SON] (BE)

Ageratella microphylla (Sch. Bip.) A. Gray ex S. Watson var. sonorana B. L. Turner [SIN, SON] (BQ) Alloispermum scabrifolium (Hook. \& Arn.) H. Rob. [DGO, JAL, NAY, SIN, SON] (BQ)

Alvordia congesta (Rose ex Hoffm.) B. L. Turner [SIN] (BE)

Bidens sambucifolia Cav. [CHIH, SIN, SON] (VAS, BE, AR) 
Vega-Aviña et al.: Endemismo Regional en la Flora del Municipio de Culiacán, Sinaloa

Apéndice. Continuación.

Egletes viscosa (L.) Less. var. dissecta Shinners [SIN] (BE, BTC)

Guardiola rotundifolia B. L. Rob. [JAL, SIN] (BQ)

Hofmeisteria sinaloensis Gentry [SIN] (BTS)

Jaumea peduncularis (Hook. \& Arn.) Oliver \& Hiern ex Oliver [CHIH, JAL, NAY, SIN, SON] (BTC, VAS)

Lagascea decipiens Hemsl. var. glandulosa (Fernald) Stuessy [DGO, JAL, NAY, SIN, SON] (BE)

Lasianthaea ceanothifolia (Willd.) K. M. Becker var. gradata (S. F. Blake) K. M. Becker [CHIH, DGO, JAL, NAY, SIN, SON] (BQ)

Lasianthaea seemannii (A. Gray) K. M. Becker [CHIH, DGO, NAY, SIN, SON] (BQ)

Melampodium rosei B. L. Rob. [DGO, JAL, NAY, SIN] (BTS, BQ)

Montanoa tomentosa Cerv. subsp. rosei (Rose ex B. L. Rob. \& Greenm.) V. A. Funk [CHIH, NAY, SIN, SON] (BTC)

Pectis stenophylla A. Gray var. puberula (Greenm.) D. J. Keil [CHIH, DGO, SIN, SON] (BE)

Perityle grandifolia Brandegee [SIN] (BTS)

Perityle microglossa Benth. var. saxosa (Brandegee) A. M. Powell [CHIH, DGO, JAL, NAY, SIN, SON] (BE)

Stenocarpha filiformis (Hemsl.) S. F. Blake [DGO, SIN] (BTC, BQ)

Stevia glandulosa Hook. \& Arn. var. glandulosa [CHIH, DGO, JAL, NAY, SIN, SON] (BQ)

Tridax tenuifolia Rose var. microcephala Rose [SIN, SON] (BE)

Trixis pterocaulis B. L. Rob. \& Greenm. [BCS, COL, JAL, NAY, SIN, SON] (BE, BTC)

Verbesina leptochaeta A. Gray [CHIH, SIN] (BTC, BQ)

Viguiera angustifolia (Hook. \& Arn.) S. F. Blake [DGO, JAL, NAY, SIN] (BQ)

Viguiera montana Rose [CHIH, SIN, SON] (BQ)

Wedelia greenmanii B. L. Turner [CHIH, JAL, SIN, SON] (BQ)

Zinnia leucoglossa S. F. Blake [NAY, SIN] (BE)

Brassicaceae

Dryopetalon runcinatum A. Gray var. laxiflorum Rollins [CHIH, SIN, SON] (BE)

Buddlejaceae

Buddleja wrightii B. L. Rob. [JAL, NAY, SIN, SON] (BE, BTC)

Cactaceae

Cephalocereus alensis (Weber) Britton \& Rose [CHIH, JAL, NAY, SIN, SON] (BE)

Echinocereus ochoterenae J. G. Ortega [DGO, SIN] (BE, BTC)

Echinocereus subinermis Salm-Dyck var. subinermis [CHIH, DGO, SIN, SON] (BE, BTC)

Ferocactus herrerae J. G. Ortega [DGO, SIN, SON] (BE)

Ferocactus schwarzii Lindsay [SIN] (BTC, BQ)

Mammillaria gueldemanniana Backeb. [CHIH, SIN, SON] (BE)

Mammillaria marksiana Krainz [DGO, SIN, SON, ZAC] (BE, BTC, BTS)

Mammillaria mazatlanensis Schum. \& Guerke [COL, JAL, NAY, SIN, SON] (BE)

Mammillaria neoschwarzeana Backeb. [SIN, SON] (BE)

Mammillaria rubida Backeb. [SIN] (BE)

Mammillaria scrippsiana (Britton \& Rose) Orcutt [JAL, NAY, SIN] (BTS)

Mammillaria tesopacensis Craig [CHIH, DGO, SIN, SON] (BE, BTC)

Opuntia rileyi J. G. Ortega [SIN] (BE, BTC)

Opuntia thurberi Engelm. var. alamosensis (Britton \& Rose) Bravo [SIN, SON] (BE)

Opuntia wilcoxii Britton \& Rose [JAL, SIN, SON] (BE, BTC)

Pereskiopsis porteri (Brandegee) Britton \& Rose [DGO, NAY, SIN, SON] (M, BE) 
Apéndice. Continuación.

Rathbunia alamosensis (J. M. Coulter) Britton \& Rose [NAY, SIN, SON] (BE, BTC)

Rathbunia kerberi (K. Schum.) Britton \& Rose [COL, DGO, JAL, NAY, SIN] (M, BE)

Stenocereus martinezii (J. G. Ortega) Bravo [SIN] (BE, BTC)

Caesalpiniaceae

Caesalpinia palmeri S. Watson [BCS, SIN, SON] (BE, BTC)

Caesalpinia sonorae Rose \& I. M. Johnst. [SIN, SON] (BE)

Chamaecrista chamaecristoides (Colladon) Greene var. brandegeei (Britton \& Rose) Irwin \& Barneby $[\mathrm{SIN}](\mathrm{BE})$

Chenopodiaceae

Atriplex barclayana (Benth.) D. Dietr. subsp. barclayana [BC, BCS, SIN, SON] (M, BE)

Convolvulaceae

Merremia palmeri (S. Watson) Hallier f. [SIN, SON] (BE)

Crassulaceae

Echeveria kimnachii Meyrán \& Vega [SIN] (BTS)

Sedum copalense Kimnach [SIN] (BTS)

Cucurbitaceae

Dieterlea fusiformis E. J. Lott [COL, JAL, SIN] (BE)

Echinopepon cirrhopedunculatus Rose [CHIH, NAY, SIN, SON] (BE)

Ibervillea maxima Lira \& Kearns [JAL, NAY, SIN] (BE)

Ibervillea sonorae (S. Watson) Greene var. sonorae [SIN, SON] (BE)

Schizocarpum palmeri Cogn. \& Rose [JAL, NAY, SIN, SON] (BE)

Cuscutaceae

Cuscuta leptantha Engelm. [BC, BCS, SIN] (BE)

Cuscuta macrocephala Schaffner [BCS, NAY, SIN] (BE)

Ebenaceae

Diospyros aequoris Standl. [JAL, NAY, SIN] (BE)

Diospyros californica (Brandegee) I. M. Johnst. var. californica [BCS, SIN, SON] (M, BE)

Diospyros sinaloensis S. F. Blake [JAL, NAY, SIN] (BE)

Euphorbiaceae

Bernardia gentryana Croizat [JAL, NAY, SIN] (BE)

Chamaesyce incerta (Brandegee) Millsp. [BCS, NAY, SIN, SON] (BE)

Cnidoscolus sinaloensis Breckon [NAY, SIN] (BE, BTC)

Croton culiacanensis Croizat [JAL, SIN] (BE)

Ditaxis sinaloae I. M. Johnst. [SIN] (BE)

Drypetes gentryi Monach. [SIN, SON] (VAS, BTS)

Euphorbia californica Benth. [BCS, COL, SIN, SON] (M, BE)

Jatropha cinerea (Ortega) Müll. Arg. [BC, BCS, DGO, SIN, SON] (M, BE)

Jatropha cordata (Ortega) Müll. Arg. [CHIH, JAL, NAY, SIN, SON] (BE)

Jatropha cordifolia (Torr.) Müll. Arg. [SIN] (BE) 
Apéndice. Continuación.

Jatropha mcvaughii Dehgan \& Webster [DGO, JAL, NAY, SIN] (BE)

Jatropha purpurea Rose \& Pax [BCS, SIN, SON] (M, BE)

Pedilanthus macrocarpus Benth. [BC, BCS, COL, JAL, NAY, SIN, SON] (BE)

Phyllanthus standleyi McVaugh [JAL, SIN] (BE, BTC)

Fabaceae

Aeschynomene petraea B. L. Rob. var. grandiflora Rudd [DGO, JAL, NAY, SIN] (BTS, BQ)

Brongniartia goldmanii Rose [NAY, SIN] (BE)

Crotalaria nayaritensis Windler [JAL, NAY, SIN] (BQ)

Dalea revoluta S. Watson [CHIH, DGO, JAL, SIN, SON] (BTC)

Dalea scandens (Mill.) Clausen var. occidentalis (Rydb.) Barneby [JAL, SIN, SON] (BE)

Dalea versicolor Zucc. var. argyrostachys (Hook. \& Arn.) Barneby [NAY, SIN] (BQ)

Erythrina lanata Rose subsp. occidentalis (Standl.) Krukoff \& Barneby [COL, JAL, NAY, SIN] (BE, BTC)

Indigofera salmoniflora Rose [NAY, SIN] (BE, BTS)

Lonchocarpus sinaloensis (Gentry) F. J. Hermann [DGO, JAL, SIN] (BE, BTC)

Marina minor (Rose) Barneby [DGO, SIN] (BTC)

Phaseolus mcvaughii A. Delgado [SIN] (BE)

Piscidia mollis Rose [SIN, SON] (BE)

Tephrosia submontana (Rose) Riley [JAL, NAY, SIN] (BE)

Fagaceae

Quercus albocincta Trel. [BCS, CHIH, SIN, SON] (BQ, BTS, BTC)

Fouquieriaceae

Fouquieria macdougalii Nash [CHIH, SIN, SON] (BE)

Lamiaceae

Salvia alamosana Rose [CHIH, DGO, NAY, SIN, SON] (BTC)

Salvia crucis Epling [DGO, SIN] (BTC, BQ)

Salvia fallax Fernald [NAY, SIN] (BE, BTC, BTS)

Salvia mazatlanensis Fernald [NAY, SIN] (BE, BTC, AR)

Salvia seemannii Fernald [CHIH, DGO, NAY, SIN, SON] (BQ)

Lennoaceae

Pholisma culiacanum (Dressler \& Kuijt) Yatskievych [SIN, SON] (BE)

Loranthaceae

Cladocolea inconspicua (Benth.) Kuijt [JAL, NAY, SIN] (BE)

Struthanthus condensatus Kuijt [COL, JAL, NAY, SIN] (BE, BTC)

Lythraceae

Cuphea crassifolia S. A. Graham [SIN] (BE)

Cuphea ferrisiae Bacig. var. rosea S. A. Graham [COL, JAL, NAY, SIN] (BE)

Malpighiaceae

Bunchosia sonorensis Rose [NAY, SIN, SON] (BE, BTC)

Malpighia umbellata Rose [SIN, SON] (BE) 
Apéndice. Continuación.

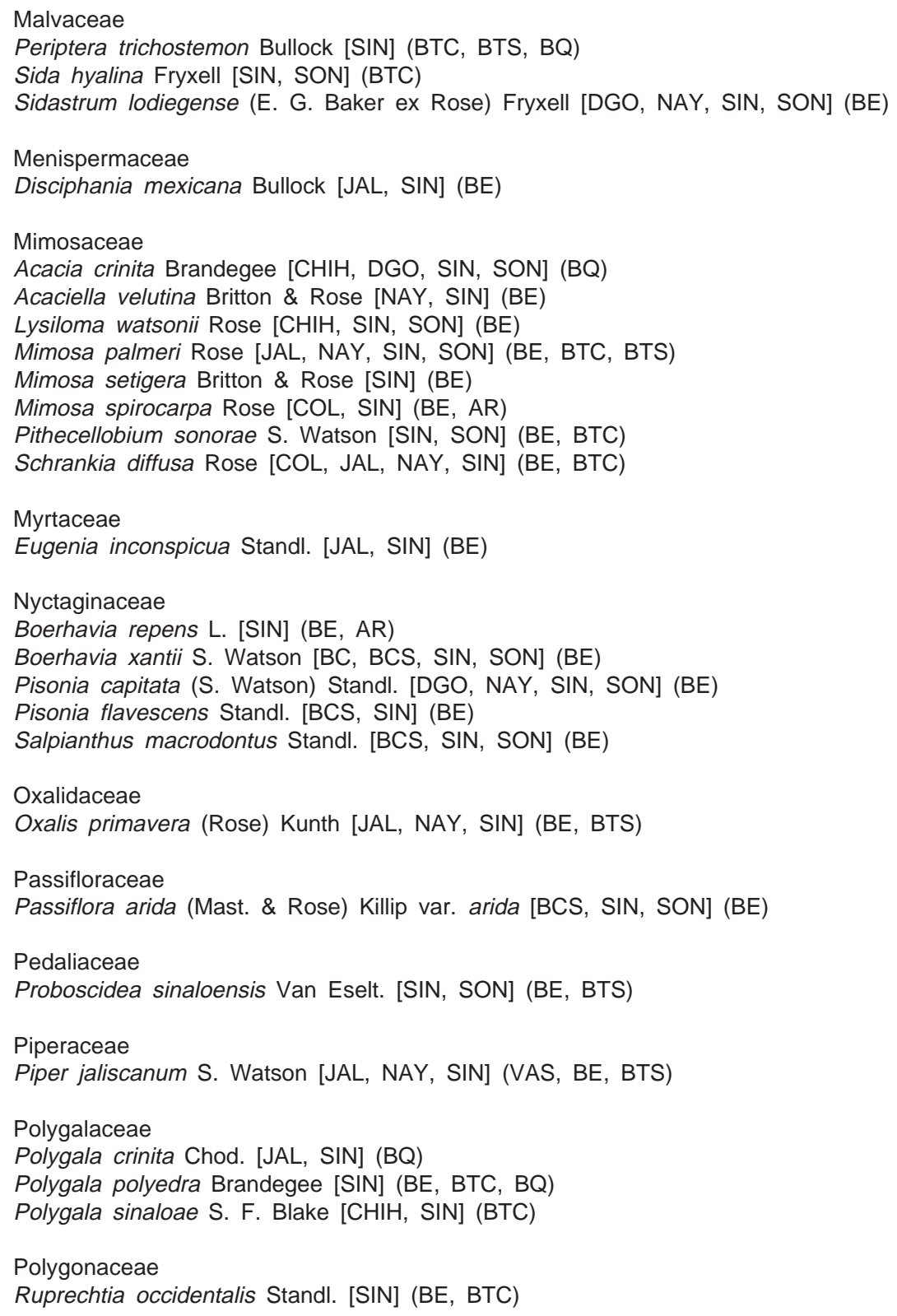


Vega-Aviña et al.: Endemismo Regional en la Flora del Municipio de Culiacán, Sinaloa

Apéndice. Continuación.

Rhamnaceae

Gouania rosei Wiggins [BC, BCS, CHIH, JAL, NAY, SIN, SON] (BE, BTC, AR)

Rubiaceae

Chiococca petrina Wiggins [CHIH, SIN, SON] (BTS)

Diodia crassifolia Benth. [NAY, SIN] (M, BE)

Guettarda filipes Standl. [DGO, NAY, SIN] (BE)

Rutaceae

Esenbeckia hartmanii B. L. Rob. \& Fernald [SIN, SON] (M, BE)

Zanthoxylum arborescens Rose [BCS, JAL, NAY, SIN, SON] (BE, BTC, BTS)

Sapindaceae

Serjania palmeri S. Watson [SIN, SON] (BE, BTC)

Sapotaceae

Sideroxylon occidentale (Hemsl.) T. D. Penn. [BCS, SIN, SON] (BE, BTC)

Sideroxylon persimile (Hemsl.) T. D. Penn. subsp. subsessiliflorum (Hemsl.) T. D. Penn. [CHIH, COL, DGO, JAL, SIN, SON] (BE)

Scrophulariaceae

Russelia tepicensis B. L. Rob. [NAY, SIN] (BE, BTC, BTS, BQ)

Schistophragma multifida (Michx.) Benth. [CHIH, SIN] (BE)

Solanaceae

Datura lanosa Barclay ex R. Bye [CHIH, NAY, SIN, SON] (BE, BTS)

Datura reburra Barclay [SIN, SON] (BE, AR)

Tiliaceae

Heliocarpus attenuatus S. Watson $[\mathrm{CHIH}, \mathrm{SIN}, \mathrm{SON}](\mathrm{BTC})$

Violaceae

Hybanthus fruticulosus (Benth.) I. M. Johnst. [BCS, SIN, SON] (BE)

Hybanthus serrulatus Standl. [JAL, SIN, SON] (BE)

Zygophyllaceae

Guaiacum coulteri A. Gray var. palmeri (Vail) I. M. Johnst. [SIN, SON] (M, BE) 